\title{
Dynamics of the superfluid to Mott-insulator transition in one dimension
}

\author{
S. R. Clark and D. Jaksch \\ Clarendon Laboratory, University of Oxford, Parks Road, Oxford OX1 3PU, United Kingdom
}

(Received 25 May 2004; published 12 October 2004)

\begin{abstract}
We numerically study the superfluid to Mott insulator transition for bosonic atoms in a one-dimensional lattice by exploiting a recently developed simulation method for strongly correlated systems. We demonstrate this method's accuracy and applicability to Bose-Hubbard model calculations by comparison with exact results for small systems. By utilizing the efficient scaling of this algorithm we then concentrate on systems of comparable size to those studied in experiments and in the presence of a magnetic trap. We investigate spatial correlations and fluctuations of the ground state as well as the nature and speed at which the superfluid component is built up when dynamically melting a Mott insulating state by ramping down the lattice potential. This is performed for slow ramping, where we find that the superfluid builds up on a time scale consistent with single-atom hopping and for rapid ramping where the buildup is much faster than can be explained by this simple mechanism. Our calculations are in remarkable agreement with the experimental results obtained by Greiner et al. [Nature (London) 415, 39 (2002)].
\end{abstract}

DOI: $10.1103 /$ PhysRevA.70.043612

PACS number(s): 03.75.Lm

\section{INTRODUCTION}

Recent experiments on loading Bose-Einstein condensates into an optical lattice have allowed for the creation and study of strongly correlated systems of atoms [1-4]. In particular the superfluid (SF) to Mott insulating (MI) transition first observed in a seminal experiment by Greiner et al. [1] has received a lot of attention since it impressively demonstrated a clean realization of the Bose-Hubbard model (BHM) [5] which has long been considered a toy model in condensed matter physics. Furthermore, in the ideal MI state each atom is localized to a lattice site corresponding to a commensurate filling of the optical lattice with zero-particle-number fluctuations. These properties make MI states attractive candidates for several applications, most notably quantum memory, quantum computing [6-12], and quantum simulations of many-body quantum systems $[13,14]$.

The BHM Hamiltonian describes atoms loaded into a sufficiently deep optical lattice $[15,16]$. It contains a kinetic energy term, with matrix element $J$, describing the hopping of particles from one site to the next and an interaction term, with matrix element $U$, which accounts for the repulsion of two atoms occupying the same site. The ratio $U / J$ increases with the depth of the optical lattice and can be varied over several orders of magnitude by tuning the optical lattice parameters [15]. In particular by changing the intensity of the laser beams creating the optical lattice it is possible to vary $J$ and $U$ on time scales much smaller than the decoherence time of the system. This opens up the possibility of directly studying the dynamics of the BHM during the quantum phase transition at temperature $T=0[1,17]$. According to mean-field (MF) theory this phase transition occurs at $u_{c}$ $=U / z J \approx 5.8$, where $z$ is the number of nearest-neighbor sites in the lattice $[5,18,19]$ and is easily accessible in an optical lattice.

In [1] the dynamics of atoms in a three-dimensional (3D) optical lattice $(z=6)$ was studied while more recently optical lattice setups where the motion of the atoms was restricted to
1D $(z=2)$ [2] were investigated. These experiments revealed some striking properties of the quantum phase transition. In particular a feature which is yet to be fully understood is the time scale over which coherence is built up throughout the atomic system when going from the MI to the SF limit [1]. Indeed it cannot be easily explained using MF theory and numerical studies of this dynamical effect were, until now, limited to small systems of approximately ten atoms. Recently, however, it has been shown that quantum computations on 1D systems of qubits which do not give rise to strong entanglement can be efficiently simulated on a classical computer via the so called time-evolving block decimation (TEBD) algorithm [20]. An immediate application of this discovery is to the simulation of the time evolution of many-body $1 \mathrm{D}$ quantum systems which are governed by a nearest-neighbor Hamiltonian [21]. The BHM is one of many important model Hamiltonians which fall into this class [22]. The simulation method is efficient for all such 1D model Hamiltonians due to a universal property of 1D systems that their ground state and lowest-lying excitations tend to contain only a small amount of entanglement [21].

In this paper we restrict our attention to the 1D BHM with our physical motivation being to study the nature and speed at which the superfluid component is built up as the system is dynamically driven through the SF-MI transition. By exploiting the efficient scaling of the TEBD algorithm with the size of the system we are able to investigate this phenomenon for setups which are of comparable size to those studied in experiments [2]. First, in Sec. II, we introduce the 1D BHM for describing atoms in optical lattices and briefly introduce the TEBD algorithm as used in this paper. In Sec. III we then demonstrate the applicability of the TEBD to the BHM by comparison with exact numerical calculations for small systems. This is then followed by an investigation of SF and MI ground states of larger lattice setups concentrating on their spatial correlations and occupation number fluctuations together with a comparison to MF results. We then study the dynamics of the MI to SF transition in Sec. IV when chang- 
ing the lattice depth on two different time scales. Most notably for rapid MI to SF ramping we find that the width of the central interference peak, as observed after releasing the atoms from the lattice, shrinks with an increasing total ramping time with the same functional dependence found in [1]. This result is discussed in Sec. IV B. Finally, we summarize our results in Sec. V.

\section{MODEL AND NUMERICAL METHOD}

In this section we introduce the BHM describing bosonic atoms in an optical lattice where the motion is restricted to 1D and give a short overview of the numerical method used in our simulations.

\section{A. Model}

By confining an ultracold bosonic gas in a 3D optical lattice with a large depth in the two orthogonal directions $y$ and $z$ it is possible to create an array of effective 1D systems in the $x$ direction $[2,10,23]$. The dynamics of these systems is governed by the external trapping and the optical lattice potential along the $x$ axis. The optical lattice then has a depth $V_{0}$ proportional to the laser intensity and a lattice period $a$ $=\lambda / 2$, where $\lambda$ is the wavelength of the laser light. The Hamiltonian describing each 1D system reduces to the 1D BHM (for details see Appendix A) [15] (taking $\hbar=1$ throughout)

$$
H=\sum_{m}-J\left(b_{m}^{\dagger} b_{m+1}+\text { H.c. }\right)-\mu_{m} b_{m}^{\dagger} b_{m}+\frac{U}{2} b_{m}^{\dagger} b_{m}^{\dagger} b_{m} b_{m},
$$

where the operators $b_{m}\left(b_{m}^{\dagger}\right)$ are bosonic destruction (creation) operators for a bosonic particle in site $m$, centered at $x_{m}=m a$, obeying the standard canonical commutation relations. The grand canonical Hamiltonian then has $\mu_{m}=\mu$ $-V_{T}\left(x_{m}\right)$ as the local chemical potential for site $m$, where $V_{T}$ is the external trapping potential. The parameters $U$ and $J$ can be determined in terms of the Wannier functions $w(x)$ as shown in Appendix A, and under the assumptions outlined are independent of the lattice site $m$ [15]. Their ratio can be varied over a wide range by dynamically changing the depth $V_{0}$ of the optical lattice. For all the systems considered here we take the wavelength of the light used to form the optical lattice as $\lambda=826 \mathrm{~nm}$, and the atomic species trapped as ${ }^{87} \mathrm{Rb}$, where $a_{s}=5.1 \mathrm{~nm}$.

\section{B. Numerical method}

In this paper we exploit the recently devised TEBD simulation algorithm $[20,21]$ which allows the dynamics of $1 D$ systems with nearest-neighbor interactions, such as the BHM, to be computed accurately and efficiently. The TEBD algorithm has been shown to be closely related to the density matrix renormalization group (DMRG) [24,25]. Over the past decade the DMRG has provided enormous insight into the static and dynamic equilibrium properties of 1D systems. Although originally devised as a ground-state method, it has been extended to yield accurate low-energy spectra [26] and also to calculations of the real time evolution of 1D systems [27] which is of particular importance here. The approach of [27] is to take the DMRG ground state $|\psi(0)\rangle$ obtained for the initial Hamiltonian and use it to define a decimation of the Hilbert space in which the Schrödinger equation is numerically integrated. The key assumption, and most severe approximation, within this scheme is that this static subspace defined by $|\psi(0)\rangle$ is adequate to approximate $|\psi(t)\rangle$ with reasonable accuracy for all times. In general this will only be true for short periods of time. Novel methods have been devised [28] which can maintain the accuracy over longer periods by "targeting" other states in addition to the ground state, but in doing so the efficiency of the computation is significantly reduced [29]. In contrast the TEBD algorithm can maintain typical DMRG accuracies while remaining efficient. Despite their differing origins it has recently been shown that TEBD and DMRG algorithms share some crucial conceptual and formal similarities [22,29]. Indeed both methods search for an approximation to the true wave function within a restricted class of wave functions which are described by matrix product states $[30,31]$ and do so with identical decomposition and truncation procedures. The essential difference, which we shall emphasize shortly, is that the TEBD algorithm updates the matrix product decomposition directly and in such a way that the resulting decimated subspace in which the time evolution is computed is optimally adapted at each step [22].

Here we briefly outline the essential features of the TEBD algorithm, with specific attention to its application to the BHM. Let us consider a 1D BHM composed of $M$ sites. An arbitrary state of this system can be expanded in the Fock basis

$$
|\psi\rangle=\sum_{n_{1}=0}^{\infty} \ldots \sum_{n_{M}=0}^{\infty} c_{n_{1} \cdots n_{M}}\left|n_{1}, \ldots, n_{M}\right\rangle,
$$

where $\left|n_{m}\right\rangle$ denotes the Fock state of $n_{m}$ particles in site $m$. For the purpose of simulating this system the number of Fock basis states per lattice site must be cut off to some upper limit $n_{\max }$. In all the numerical calculations we performed $n_{\max }=5$. This is sufficient to avoid any cutoff effects in the bosonic occupation, as long as only small filling factors of the lattice are used and the on-site interaction energy $U$ is sufficiently large compared to the hopping energy $J$.

Now suppose we split the system into two contiguous parts $A_{m}$ composed of the first $m$ sites and $B_{m}$ composed of the last $M-m$ sites. We can think of this partitioning as cutting the $m$ th bond situated between sites $m$ and $m+1$. For any state $|\psi\rangle$ a Schmidt decomposition (SD) can be performed which renders the state in the form

$$
|\psi\rangle=\sum_{\alpha=1}^{\chi_{m}} \lambda_{\alpha}^{[m]}\left|\phi_{\alpha}^{A_{m}}\right\rangle\left|\phi_{\alpha}^{B_{m}}\right\rangle,
$$

where $\chi_{m}$ is the Schmidt rank of the SD, $\lambda_{\alpha}^{[m]}$ are the Schmidt coefficients, and $\left|\phi_{\alpha}^{c}\right\rangle$, with $c \in\left\{A_{m}, B_{m}\right\}$, are the corresponding Schmidt states of the respective subsystems. The Schmidt rank $\chi_{m}$ is a useful measure of the entanglement between the two subsystems $A_{m}$ and $B_{m}$ [20]. Given any state 
(a)
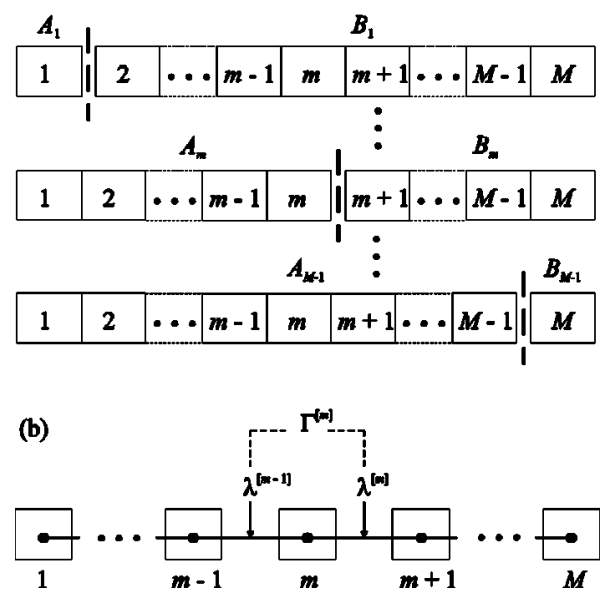

FIG. 1. (a) The sequence of contiguous partitions of the system in which the SD are computed. The coefficients and states from these SD are then used to form the $\Gamma$ and $\lambda$ tensors. (b) A depiction of the $\Gamma$ tensors associated to lattice sites and $\lambda$ tensors associated to bonds between those sites.

$|\psi\rangle$ a set of $(M-1)$ SD can be performed according to a sequence of such partitions of the system with $m \in\{1 \cdots M$ $-1\}$, as depicted in Fig. 1(a).

Using the $\lambda_{\alpha}^{[m]}$ and states $\left|\phi_{\alpha}^{s}\right\rangle$ for each subsystem obtained from these SD it is possible [20] to construct a set of $\Gamma$ and $\lambda$ tensors which are equivalent to a matrix product decomposition of the expansion coefficients $c_{n_{1} \cdots n_{M}}$ of $|\psi\rangle$ in the fixed Fock basis [22]. Specifically one finds

$$
c_{n_{1} \cdots n_{M}}=\sum_{\alpha_{1}, \ldots, \alpha_{M-1}} \Gamma_{\alpha_{1}}^{[1] n_{1}} \lambda_{\alpha_{1}}^{[1]} \Gamma_{\alpha_{1} \alpha_{2}}^{[2] n_{2}} \lambda_{\alpha_{2}}^{[2]} \cdots \lambda_{\alpha_{M-1}}^{[M-1]} \Gamma_{\alpha_{M}}^{[1] n_{M}},
$$

where $n_{m}$ is the occupation number of site $m$, and $\alpha_{m}$ are the Schmidt indices of the $m$ th partition, each of which sums from 1 to its respective Schmidt rank $\chi_{m}$. With reference to Fig. 1(b) we note that each $\lambda_{\alpha_{m}}^{[m]}$ is labeled by the bond between sites $m$ and $m+1$, along with the corresponding Schmidt index $\alpha_{m}$, whereas each $\Gamma_{\alpha_{m-1} \alpha_{m}}^{[m] n_{m}}$ is labeled by a site $m$ which resides between the two bonds $m-1$ and $m$, and so also possess the Schmidt indices $\alpha_{m-1}$ and $\alpha_{m}$ of these bonds.

Under the circumstances described the expansion, Eq. (4), is exact and as such the number of parameters stored could grow exponentially with the size of the system. However, it is a general feature of $1 \mathrm{D}$ systems with nearest-neighbor interactions that the entanglement within their ground state and low-lying excitations depends weakly on the size of the system [21]. Indeed it can be shown that the entanglement of a block of size $\ell$ with the rest of the system remains finite as $\ell \rightarrow \infty$ in 1D systems or at worst grows logarithmically with $\ell$ at criticality [22]. Consequently the entanglement between the blocks of any of the $(M-1)$ SD illustrated in Fig. 1(a) can be saturated by some fixed Schmidt rank, which for the systems we consider is typically small. It is this fact that accounts for the success of the DMRG in 1D systems. Similarly within TEBD it allows the maximum possible Schmidt rank used in the matrix product decomposition, Eq. (4), to be fixed to some value $\chi$, thereby truncating it to the most significant contributions. For an appropriate choice of $\chi$ this approximation will be accurate with the error proportional to the sum of the discarded eigenvalues in the SD [21]. This clear interpretation of the central numerical parameter $\chi$ within TEBD is very useful. Once a value of $\chi$ is found to saturate the entanglement of the ground state and low-lying excitations of a system then this a direct measure of the role of entanglement in the dynamics of the system. In total the scaling in the number of parameters within the expansion, Eq. (4), is quadratic in $\chi$ and linear in the size of the system $M$ and in $n_{\max }$. So upon fixing $\chi$ and thus preventing its possible exponential dependence on $M$, the description becomes efficient. As with DMRG this decomposition of a state generates, for all practical purposes, an optimal $\chi \times \chi$ matrix product state [22]. A noteworthy limit of this is the approximation where $\chi=1$, which forces the description of the system to be of product form with respect to all sites. Using the TEBD algorithm under this severe restriction is in fact equivalent to MF theory and the Gutzwiller ansatz $[5,18,32,33,17]$.

Another crucial advantage of the TEBD algorithm is that once a state is expressed in the matrix product form, Eq. (4), one- and two-site unitary transformations can be applied directly and exactly to the system such that the resulting state can be efficiently returned to a matrix product form [22]. Indeed, given a partitioning of the system into a two-blocktwo-site configuration $[1 \cdots m-1][m m+1][m+2 \cdots M]$, the application of a two-site unitary to sites $m$ and $m+1$ only requires updates to be performed on the tensors local to those sites-namely, $\Gamma_{\alpha_{m-1} \alpha_{m}}^{[m] n_{m}} \lambda_{\alpha_{m}}^{[m]} \Gamma_{\alpha_{m} \alpha_{m+1}}^{[m+1] n_{m+1}}$. The major computational effort of this update is limited to the rediagonalization of the reduced density matrix of one of the adjacent site and block subsystems, such as sites $[m+1][m+2 \cdots M]$, which is of dimension $\left(\chi n_{\max }\right) \times\left(\chi n_{\max }\right)$ at most [20]. The crucial feature here is that a DMRG-style truncation to only the most relevant eigenstates of this reduced density matrix occurs in an optimal way at each application of a two-site unitary. This is in contrast with time-dependent DMRG methods where the basis states which make up the matrix product decomposition are fixed at the start [22,27]. The number of basic operations required to perform this update scales as $O\left(\chi^{3}\right)$ [20].

To compute the action of the time-evolution unitary $\exp (-i H \delta t)$, for a time step $\delta t$, we first make the observation that for Hamiltonians with nearest-neighbor interactions, which are composed of two-site operators at most, terms can be separated into a sum of those involving odd sites, $F$, and those involving even sites, $G$ :

$$
\begin{gathered}
F=\sum_{n \text { odd }} F_{n, n+1}, \\
G=\sum_{n \text { even }} G_{n, n+1}, \\
H=F+G .
\end{gathered}
$$

Given that no terms within $F$ involve the same lattice sites they all commute amongst themselves. Thus the action of $\exp (-i F \delta t)$ can be computed exactly as 


$$
e^{-i F \delta t}=\prod_{n \text { odd }} e^{-i F_{n, n+1} \delta t}
$$

Since each term in this product is a two-site unitary, they can be applied individually to the state with the method detailed in [20], and the same is also true for $G$. The complications in computing the time evolution arise from the fact that $F$ and $G$ do not in general commute, and hence we approximate the unitary time-evolution operator $\exp [i(F+G) \delta t]$ using a Trotter expansion. Ignoring their noncommutativity would constitute a first order expansion. If we define

$$
s_{2}(F, G, y)=e^{-i F y / 2} e^{-i G y} e^{-i F y / 2},
$$

then the second-order expansion follows when $y=\delta t$. For the numerical simulations performed in this paper the fourthorder expansion [34] was used, which has the form

$$
e^{-i(F+G) \delta t}=\prod_{l=1}^{5} s_{2}\left(F, G, q_{l} \delta t\right)+O\left(\delta t^{5}\right),
$$

where the parameters $q_{l}$ are defined as

$$
q_{1}=q_{2}=q_{4}=q_{5} \equiv q=\frac{1}{\left(4-4^{1 / 3}\right)}, \quad q_{3}=1-4 q .
$$

A detailed analysis of the errors and computational cost of TEBD is given in [21], where it is shown that the Trotter error propagates quadratically with the simulated time and so the accuracy of the method can be maintained for long periods with appropriate choices of the parameters.

The pure TEBD implementation we employ here can be improved further by combining the advantageous features of TEBD outlined with the well-established optimizations of DMRG such as good quantum numbers and White's "state prediction" method. In doing so an adaptive time-dependent DMRG algorithm is obtained [22,29] illustrating the extremely close relationship between these two methods. Finally we note the very recent advances in generalizing TEBD and DMRG to describe mixed state dynamics and generic master equation evolution of 1D systems with nearestneighbor coupling [35,36]. This opens up the possibility of simulating finite temperature effects, decoherence, and dissipation.

\section{GROUND STATES OF THE BHM}

We first investigate the ground state of the BHM and compare the numerical results with the exact ground states for a small homogeneous system. Then we consider a larger system in the presence of a shallow magnetic trap $V_{T}$ superimposed on the lattice and compare the results to those predicted by MF theory. In all cases the numerical ground state was computed with the TEBD algorithm using continuous imaginary time evolution from a simple product state, as detailed in [21].

\section{A. Comparison of exact and simulated ground states}

To investigate the accuracy of the numerical simulation and its applicability to the BHM we first consider a small
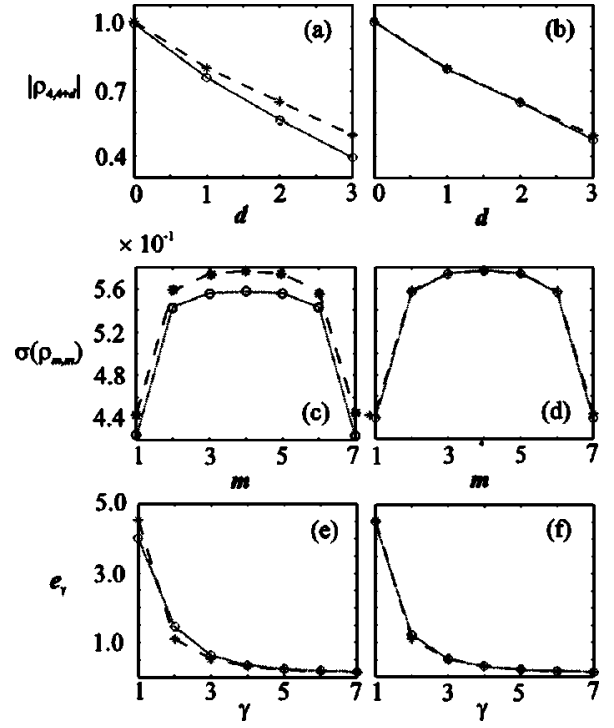

FIG. 2. Comparisons of the numerical $(\bigcirc)$ and exact $(*)$ calculations with $U / 2 J=2$ for spatial correlations $\left|\rho_{4,4+d}\right|$ with the central site $m=4$ obtained for (a) $\chi=3$ and (b) $\chi=5$, the standard deviation of the site occupation $\sigma\left(\rho_{m, m}\right)$ obtained for (c) $\chi=3$ and (d) $\chi=5$, and the spectrum $e_{\gamma}$ of the one-particle density matrix obtained for (e) $\chi=3$ and (f) $\chi=5$. The dashed and dotted curves shown are to guide the eye.

system in which an exact solution can be found readily. Specifically we use an optical lattice composed of $M=7$ sites, a trapping potential of $V_{T}=0$ with box boundary conditions, and a total number of particles $N=7$. The ground state is then calculated numerically and exactly for $U / 2 J=2,6$, and 20, corresponding to the SF, intermediate, and MI regimes, respectively. The numerical simulation was performed for $\chi$ $=3,5$, and 7 in each case.

The one-particle density matrices $\rho_{m, n}=\left\langle b_{m}^{\dagger} b_{n}\right\rangle$ obtained for each regime for the numerical and exact calculations are visually indistinguishable in all cases. In order to highlight the extent of the agreement we present a number of other plots. Specifically in the SF regime the comparisons of the spatial correlation of the central site $\left|\rho_{4,4+d}\right|$ as a function of the distance $d$ are shown in Figs. 2(a) and 2(b) between the exact and numerical calculations using $\chi=3$ and $\chi=5$. Identical comparisons of the standard deviation of the site occupation $\sigma\left(\rho_{m, m}\right)=\left(\left\langle N_{m}^{2}\right\rangle-\left\langle N_{m}\right\rangle^{2}\right)^{1 / 2}$, where $N_{m}=b_{m}^{\dagger} b_{m}$, and the spectrum of the one-particle density matrices $e_{\gamma}$ [normalized with $\operatorname{tr}(\rho)=N]$ are shown in Figs. 2(c), 2(d) and 2(e), 2(f), respectively. These results show that although there is qualitative agreement between exact and $\chi=3$ calculation, almost all expectation values have a maximum deviation from the exact calculation improved by an order of magnitude with $\chi=5$. As expected for a SF ground state the one-particle density matrix spectrum in Fig. 2(f) is dominated by one eigenvalue of $\operatorname{order} N$. However, given that the lattice still has a nonzero depth this state deviates from that of a pure SF, where $\left|\psi_{\mathrm{SF}}\right\rangle \propto\left(\Sigma_{m} b_{m}^{\dagger}\right)^{N}|\mathrm{vac}\rangle$, since the sum of the remaining eigenvalues (in descending order) is $\sum_{\gamma=2}^{7} e_{\gamma} \approx 2.5$ and so represents a significant quantum depletion of the SF.

For the intermediate and MI regime a similar factor of improvement can be obtained; however, in this case the $\chi$ 


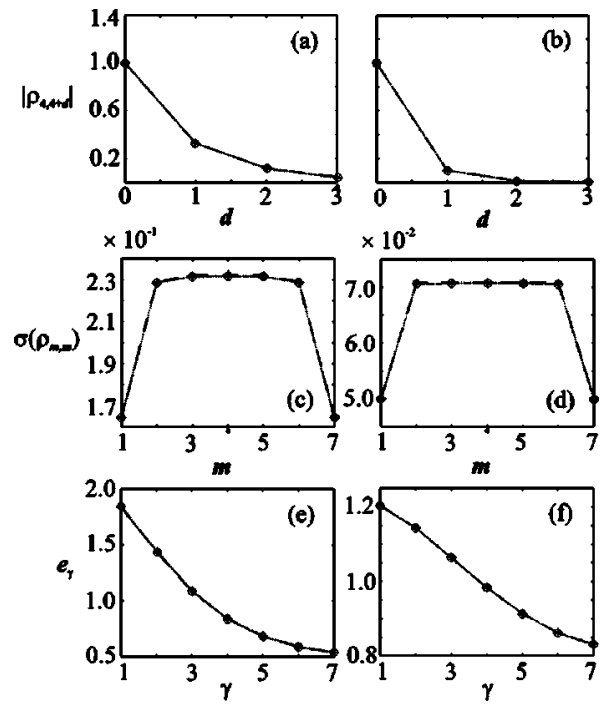

FIG. 3. Comparisons of the numerical $(\bigcirc)$ and exact $(*)$ calculations, where the numerics were all performed with $\chi=3$, for spatial correlations $\left|\rho_{4,4+d}\right|$ with the central site $m=4$ obtained with (a) $U / 2 J=6$ and (b) $U / 2 J=20$, the standard deviation of the site occupation $\sigma\left(\rho_{m, m}\right)$ obtained with (c) $U / 2 J=6$ and (d) $U / 2 J=20$, and the spectrum $e_{\gamma}$ of the one-particle density matrix obtained with (e) $U / 2 J=6$ and (f) $U / 2 J=20$. Note the differing scales and that the dashed and dotted curves are shown only to guide the eye.

$=3$ calculation already yields excellent agreement with the exact calculation. Specifically we find the infidelity between the numerical and exact many-body state is $1-F=1$ $-\left|\left\langle\psi_{0} \mid \psi_{0}^{\prime}\right\rangle\right| \approx 10^{-5}$, where $\left|\psi_{0}\right\rangle\left(\left|\psi_{0}^{\prime}\right\rangle\right)$ is the numerical (exact) ground state and the temperature corresponding to the difference in their ground-state energy as $\Delta T \approx 10^{-2} \mathrm{nK}$. The comparisons of the spatial correlation, site occupancy standard deviation, and one-particle density matrix spectrum for $U / 2 J=6$ and $U / 2 J=20$, with $\chi=3$, are shown in Fig. 3 .

These plots, along with those where $\chi=5$ for the SF regime, illustrate the onset of increasing MI characteristics in the ground states, in particular the rapidly decreasing spatial correlations and fluctuations in occupancy, as well as the change in the spectrum from being dominated by one singleparticle state to having seven almost equally occupied orbitals. These indicate that the MI ground state obtained is a very close approximation to that of a pure MI, where $\left|\psi_{\mathrm{MI}}\right\rangle$ $\propto \prod_{m} b_{m}^{\dagger} \mid$ vac $\rangle$, representing commensurate filling of the lattice. However, given that the lattice is not infinitely deep, deviations with this pure MI state exist and are evident from the persistence of small off-diagonal correlations visible at $d=1$ in Fig. 3(b) and the spread of the spectrum about unity in Fig. 3(f).

As expected we find that the agreement between the numerical and exact calculations for a given value of $\chi$ improves with increasing $U / 2 J$ in line with the decrease in off-diagonal correlations. In all cases the $\chi=7$ results gave excellent agreement with the exact calculation. The worst case being in the SF regime where an infidelity of $1-F$ $\approx 10^{-4}$, and a deviation in ground-state energy of $\Delta T$ $\approx 10^{-2} \mathrm{nK}$ was obtained.

We note here that the exact calculation was performed with the canonical Hamiltonian with $N=7$, whereas the nu- merical simulations used the grand canonical Hamiltonian. With an appropriate choice of the chemical potential $\mu$ the average particle number can be fixed to $N=7$, enabling the comparisons above. Indeed for the calculations performed the worst absolute value of the projection of the simulated state outside the $N=7$ Fock subspace was $\left\langle\psi_{0}\left|\mathcal{P}_{N \neq 7}\right| \psi_{0}\right\rangle$ $\approx 10^{-13}$. Hence our results confirm the agreement of these approaches for small systems, and we assume the agreement holds for the larger systems.

\section{B. MI and SF states with a superimposed magnetic trap}

To consider systems closer to those studied in experiments [2] we use a lattice with $M=49$ sites and made the system inhomogeneous by superimposing a harmonic trap potential $V_{T}\left(x_{m}\right)=m_{A} \omega^{2} x_{m}^{2} / 2$, where $\omega$ is the trapping frequency and $m_{A}$ is the mass of an atom. As with the smaller system the ground states for the SF, intermediate and MI regimes were calculated. The lattice was loaded with a total number of particles $N=40$ by choosing an appropriate chemical potential $\mu$ in each regime. For all cases a trapping frequency of $\omega=2 \pi \times 70 \mathrm{~Hz}$ was used and found to be sufficient in eliminating any occupation at the boundaries of the system.

The inhomogeneity caused by a spatially varying confining potential can result in the coexistence of spatially separated SF and MI regions. Such properties have been confirmed experimentally [1,2] and have received intense theoretical study with numerical calculations, in particular through Gutzwiller-ansatz and MF theory [15,37] and quantum Monte Carlo (QMC) [38,39] and DMRG [40] simulations in 1D, as well as calculations using the QMC [41,42] and numerical renormalization group [43] methods for 2D and $3 \mathrm{D}$ systems. Here we explore the SF-MI coexistence features of BHM ground states in order to confirm the physical picture arising from our numerical calculation for the large inhomogeneous system. Specifically we make comparisons of the mean site occupancy and its standard deviation between the numerical results and those obtained by MF theory for each regime (see Appendix B for details of the MF calculation) $[33,37]$.

The one-particle density matrix of the resulting SF ground state is shown in Fig. 4(a). Important features of this state are outlined in Figs. 5(a)-5(d), where it can be seen that the system is entirely SF. The MF results for the site occupancy and its standard deviation are also shown in Figs. 5(a) and 5(b), and as expected there is good agreement between the curves in both cases. For the intermediate regime, whose one-particle density matrix is shown in Fig. 4(b), we see a system possessing alternating regions of coherent SF and incoherent MI phases [38,40]. The pattern of these regions starts with a central SF region with a mean occupancy exceeding unity, which then becomes a singly occupied MI, a SF region with mean occupancy less than unity, and finally the vacuum MI. In Figs. 5(e) and 5(f) we see that the MI region in Fig. 5(e) coincides with suppressed fluctuations in occupancy shown in Fig. 5(f). The MF curves also plotted show general agreement with these phase identifications; however, the MF curve in Fig. 5(f) predicts a significantly 

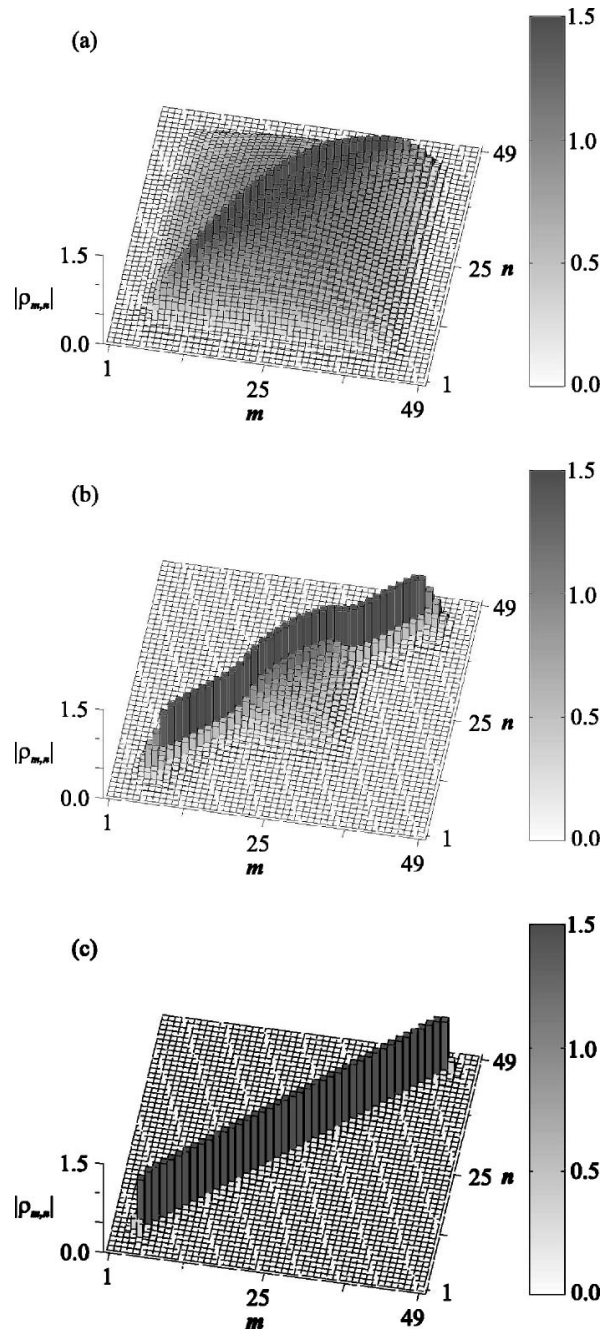

FIG. 4. The absolute value $\left|\rho_{m, n}\right|$ of the one-particle density matrix as a function of site indices $m, n$ for (a) $U / 2 J=2$, (b) $U / 2 J=6$, and (c) $U / 2 J=20$.

greater suppression of the particle number fluctuations for the MI regions than the numerical results. Such deviations are consistent with the fact that MF theory predicts a sharp and well-pronounced SF-MI phase transition [33,37]. While these prediction are known to be accurate for infinite homogeneous systems, for small inhomogeneous systems we see that the role of correlations is important and that the transition between the SF and MI regions is not established with such definiteness.

Given that the ground state for the intermediate regime exhibits significantly sized SF regions which are separated by a MI, we investigated whether any correlations were present between these regions. Evidence of such correlations would be the presence of elongated peaks in the one-particle density matrix at the off-diagonal locations corresponding to the intersection of the rows $m$ and columns $n$ of the SF regions. To within the accuracy obtained with $\chi=5$ no such correlation peaks were found in the one-particle density matrix of the ground state. This was confirmed with continuous imaginary time evolution not only for a product initial state used conventionally, but also with an initial state which al-
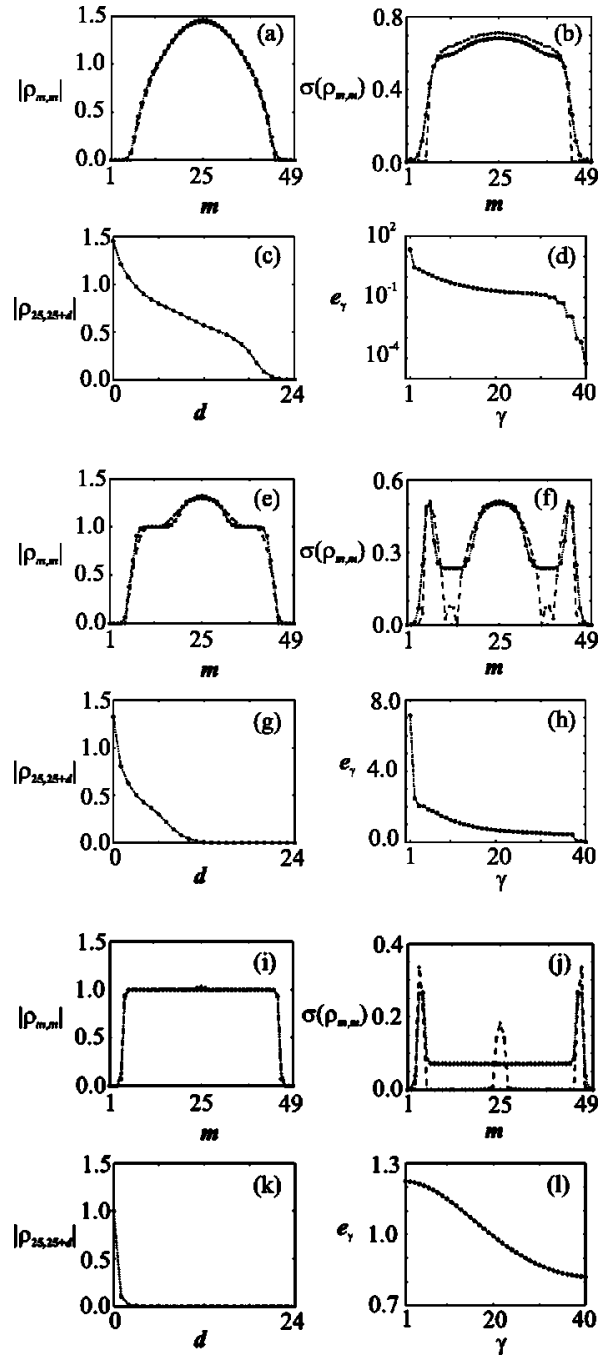

FIG. 5. Specific plots for the three regimes. For $U / 2 J=2$ there is (a) the site occupancy $\left|\rho_{m, m}\right|$, (b) the standard deviation of the site occupancy $\sigma\left(\rho_{m, m}\right)$, both with the MF calculation shown as the dashed curve, (c) the spatial correlations from the central site $m$ $=25$, and (d) the spectrum $e_{\gamma}$ of the one-particle density matrix, showing only the 40 nonzero eigenvalues. The same plots are presented for $U / 2 J=6$ in (e)-(h) and for $U / 2 J=20$ in (i)-(1).

ready contained significant correlations. Our results suggest that if such SF correlations do exist within the ground state, then they are extremely fragile. Despite this we shall see shortly that such correlations do occur readily in dynamical situations which cross the MI-SF transition, where the system does not necessarily remain in the ground state.

Lastly, the one-particle density matrix for the MI regime is shown in Fig. 4(c). It is clear from this and the corresponding plots in Figs. 5(i) and 5(j) that the system is almost completely in the singly occupied MI phase, aside from the small SF regions at the far extremes before the vacuum. Their presence is typified by the two peaks in the occupancy standard deviation shown in Fig. 5(j). The MF calculation in this case gives the same identification of the regions, except for the very center of the trap, where a small SF region is predicted to exist, as evidenced by the central peak in the MF occupancy standard deviation curve of Fig. 5(j). Again MF 
theory predicts a much greater suppression of occupancy fluctuations in the MI regions of the system than the numerical calculation. As with the smaller system the plots of Fig. 5 for the three ground states illustrate the transition from predominately SF to MI characteristics.

In all cases the ground-state calculations were performed with $\chi=5$. To ensure convergence the calculations were repeated for $\chi=7$. The largest deviation between these two calculations was found in the SF regime where the estimated ground-state energy differed by a temperature $\Delta T_{\chi=5 \rightarrow 7}$ $\approx 0.2 \mathrm{nK}$. We made a similar comparison between the $\chi=5$ results and those of MF theory, which are equivalent to $\chi$ $=1$, where the largest deviation was found to be $\Delta T_{\chi=1 \rightarrow 5}$ $\approx 3 \mathrm{nK}$. Given the larger occupancy of the system the calculations were also repeated with larger values of $n_{\max }$, confirming that no cutoff effects were encountered.

\section{DYNAMICS OF THE BHM}

The most novel feature of the TEBD algorithm is its capacity to efficiently simulate the dynamics of $1 \mathrm{D}$ systems which are inaccessible to exact calculation. Here we consider dynamics which are generated by varying the optical lattice depth $V_{0}(t)$ according to some ramping profile in time. The result of this is that the parameters $J(t)$ and $U(t)$ in the BHM Hamiltonian of Eq. (1) become time dependent. By appropriately choosing the range of values covered by the optical depth $V_{0}(t)$ it is possible to dynamically drive the system through the SF-MI transition. We shall consider such dynamics occurring on two different time scales: first, via a slow and smooth profile, and second, as fast linear ramping. Our objective in both cases is to observe the nature and speed in which coherence is reestablished within the system.

\section{A. Slow dynamics}

\section{Profile of the slow dynamics}

For the slow dynamical profile a smoothed "box" function was used for the depth $V_{0}(t)$. Such a profile for the dynamics has been considered before for small systems [17]. It has the form

$$
V_{0}(t)=V_{\mathrm{SF}}+\mathcal{N} \frac{V_{\mathrm{MI}}-V_{\mathrm{SF}}}{1+e^{\left[\left(t-t_{c}\right)^{2}-t_{w}^{2}\right] / t_{s}^{2}}},
$$

where $t_{c}, t_{w}$, and $t_{s}$ are time parameters specifying the center, width, and step size of the profile, respectively, while $\mathcal{N}=1$ $+e^{-t_{w}^{2} / t_{s}^{2}}$ is the scaling factor required to ensure that the depth varies from $V_{\mathrm{SF}}$ to $V_{\mathrm{MI}}$. The lattice depths $V_{\mathrm{SF}}$ and $V_{\mathrm{MI}}$ were chosen to be the depths equivalent to $U / 2 J=2$ and $U / 2 J$ $=20$, respectively, in correspondence with the parameters used in the previous section for the SF and MI regime. The exact shape of the profile for $U / 2 J$ resulting from the ramping of $V_{0}(t)$ chosen is shown in Fig. 6(a). Large time parameters have been used in order to keep the time evolution of the system sufficiently adiabatic and to prevent excessive excitations.
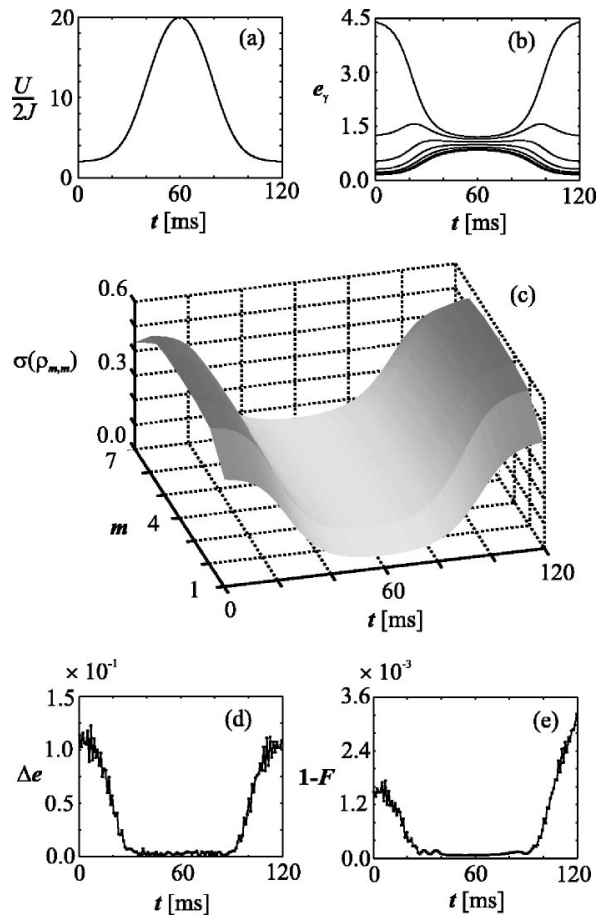

FIG. 6. Slow dynamics of the small system: (a) the resulting ramping profile of the parameter $U / 2 J$ with time, where the time parameters for the $V_{0}(t)$ profile are $t_{c}=60 \mathrm{~ms}, t_{w}=24 \mathrm{~ms}$, and $t_{s}$ $=18 \mathrm{~ms}$, (b) the spectrum of the one-particle density matrix $e_{\gamma}$ with time obtained from the numerical calculation, (c) the standard deviation of the site occupancy $\sigma\left(\rho_{m, m}\right)$ with time obtained from the numerical calculation, (d) the maximum deviation between the numerical and exact spectrum $\Delta e$ with time, and (e) the infidelity 1 $-F$ of the numerical many-body state compared to the exact state with time.

\section{Slow dynamics of the small system: $M=7$}

First, we consider the slow dynamics applied to the small system. This provides the opportunity to solve the BHM dynamics both numerically and exactly, allowing a direct comparison of the accuracy and a demonstration of the applicability of the algorithm to the dynamics of the BHM. The system was initially prepared in the SF ground state computed earlier in Sec. III A and using $\chi=5$ for the numerical calculations. The time evolution was then performed for a total time $t_{\text {tot }}=2 t_{c}$, with time $t$ running over the interval $\left[0, t_{\text {tot }}\right]$. The spectrum $e_{\gamma}$ of the one-particle density matrix $\rho_{m, n}(t)$ is plotted as a function of time in Fig. 6(b). For times $t$ where $U / 2 J<u_{c}$ the spectrum is, as expected, dominated by one large eigenvalue whose value is of order of the number of atoms. As $U / 2 J$ crosses the MF critical value $u_{c}$ the eigenvalues are found to converge around the region of 1 . Indeed the state of the system given by the numerical calculation at the time $t=t_{c}$ in the dynamics, which corresponds to $U / 2 J=20$, is found to have an infidelity with the numerical MI ground state computed earlier in Sec. III A as 1 $-F<10^{-4}$. This confirms that for a small and homogeneous system the ramping is sufficiently adiabatic to ensure that the system has entered the MI regime as the ground state and the one-particle density matrix is diagonal. 
With decreasing optical depth and, in turn, decreasing $U / 2 J$, the $\mathrm{SF}$ ground state is restored when $U / 2 J=2$ is reached again at $t=2 t_{c}$. The infidelity between the initial numerical SF ground state and the final numerical SF state was found to be $1-F<10^{-3}$. In Fig. 6(c) the behavior of the fluctuations in the site occupancy is as expected; namely, the standard deviation in site occupancy is suppressed with increasing lattice depth and restored with its subsequent decrease.

To test the accuracy of the TEBD algorithm a number of comparisons to the exact calculation were made. The simplest of these was the maximum relative deviation between the exact and numerical results for the one-particle density matrix spectrum $\Delta e=\max _{\gamma}\left(\left|1-e_{\gamma} / e_{\gamma}^{\prime}\right|\right)$, where $e_{\gamma}$ and $e_{\gamma}^{\prime}$ are the numerical and exact results, respectively. The time profile $\Delta e$ is plotted in Fig. 6(d). It is found that over the whole time evolution the relative deviation is at most $\Delta e \approx 10^{-1}$. A similar relative deviation can be defined for the standard deviation of the occupancy as $\Delta \sigma=\max _{m}\left(\left|1-\sigma_{m} / \sigma_{m}^{\prime}\right|\right)$, where it is found that $\Delta \sigma \approx 10^{-2}$ at most during the time evolution.

The most conclusive comparison, however, is the infidelity $1-F(t)$ of the exact and numerical many-body states over the time evolution, shown in Fig. 6(e). It is clear from this that the infidelity is bounded as $1-F(t)<4 \times 10^{-3}$ over the whole evolution. The shape of the infidelity profile also gives important information about the TEBD method. Namely, it fits the general observation made in Sec. III A for the ground-state calculations that for fixed numerical parameters $\chi$ and $n_{\max }$ the simulation is more accurate in the intermediate and MI regimes than the SF regime. This behavior is precisely exhibited in the time dependence of the above comparisons where significant reductions in the deviations are seen when the system enters the MI regime.

\section{Slow dynamics of the larger system: $M=49$}

The larger system possesses many of the essential characteristics of current experimental implementations of the SF-MI transition [1,2]. In particular, these include the inhomogeneous nature of the system caused by a trapping potential, and the larger number of both lattice sites and atoms, as compared with the smaller system. With a linear size of $M$ $=49$ sites the system considered is on the same scale as experiments already performed [1,2]. The major difference is that the mean occupancy at the center of our system is $\left\langle n_{c}\right\rangle$ $\approx 1.5$, roughly half that of most experiments, where it is usual to have $\left\langle n_{c}\right\rangle \approx 2.5$. While the mean occupancy undoubtedly has an important influence in the dynamics, the system simulated here is sufficiently close that it can demonstrate much of the important physics.

The slow ramping profile, Fig. 6(a), was performed identically on the larger inhomogeneous system using the ground state, Fig. 4(a), computed earlier as the initial state and for a total time $t_{\text {tot }}=3 t_{c}$. The resulting spectrum $e_{\gamma}$ of the oneparticle density matrix is plotted as a function of time in Fig. 7. General features of this spectrum follow from the smaller homogeneous system; namely, the trend for the eigenvalues to decrease as the bottom of the ramping is reached. While most eigenvalues converge to unity, as with the smaller sys-
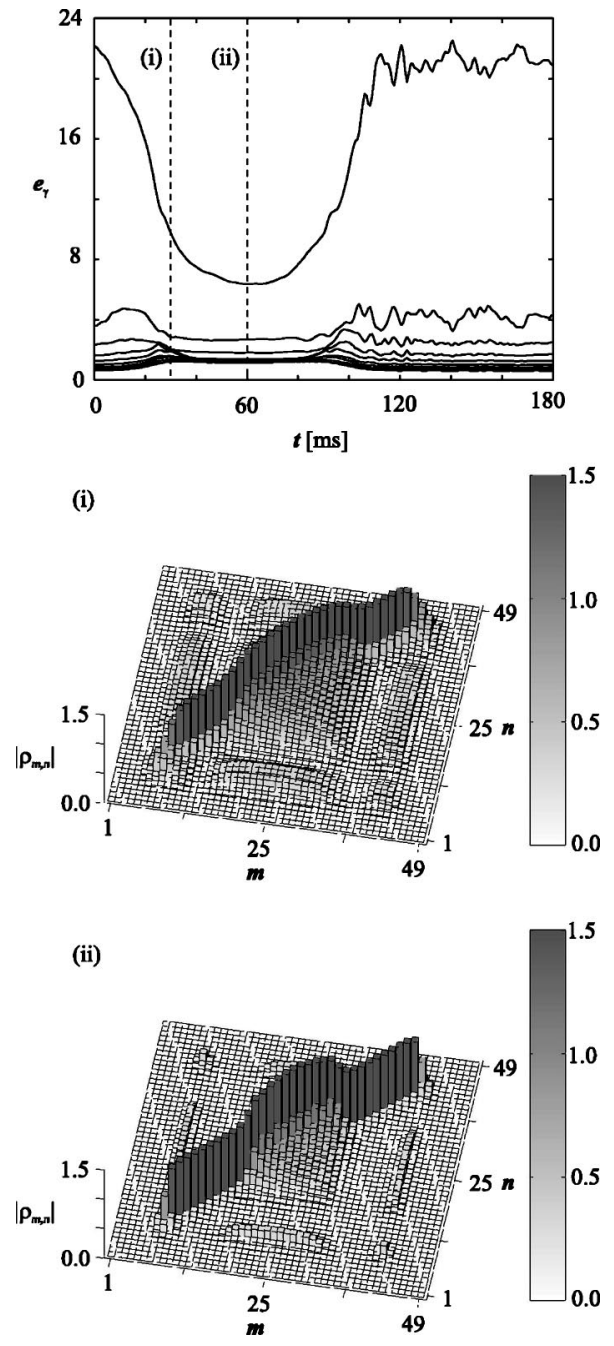

FIG. 7. The spectrum of the one-particle density matrix $e_{\gamma}$ for the slow dynamics of the larger inhomogeneous system, showing only the largest ten eigenvalues. The dashed lines denote the two times (i) $t=t_{c} / 2$ and (ii) $t=t_{c}$ for which the absolute value $\left|\rho_{m, n}\right|$ of the one-particle density matrix is plotted.

tem, one in particular can be seen to remain much larger than this during the entire dynamics. This is a clear indicator of a significant SF region within the state as it is dynamically driven into the MI regime. For the larger inhomogeneous system we see that the slow ramping profile is not adiabatic enough to bring the system into the MI ground state shown in Fig. 4(c).

Indeed to examine the nature of the state generated by the dynamics additional plots of the one-particle density matrix are also shown in Fig. 7 for the two times indicated during the dynamics. These show that the state of the system remains close in form to that of the ground state for the intermediate regime shown in Fig. 4(b), where a large SF region exists at the center of the trap. However, unlike that ground state we see that sizable correlations between the separated SF regions, which were alluded to earlier in Sec. III B, do exist and remain present even at the bottom of the profile for Fig. 7(ii) where $U / 2 J=20$.

Another important difference between the spectra of the small and larger system is the more prominent excitations 

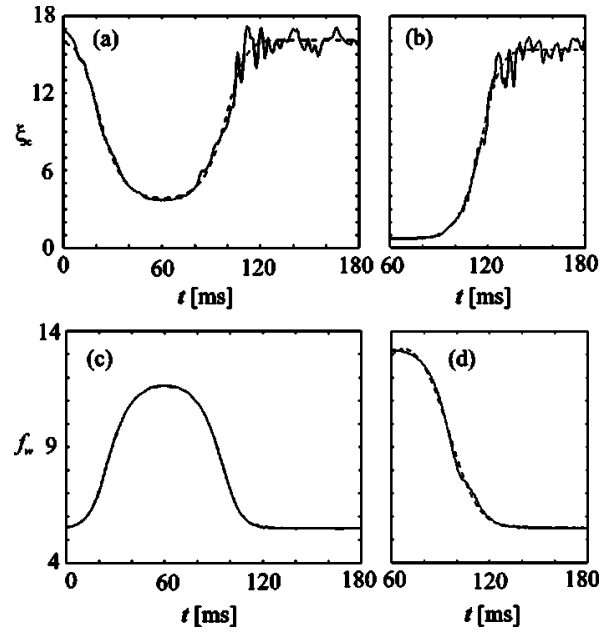

FIG. 8. Slow dynamics of the larger inhomogeneous system with the variation in the correlation cutoff $\xi_{c}$, measured in lattice sites, for (a) the complete slow ramping profile starting from the SF ground state and for (b) the MI ground state beginning at the bottom of the ramping. The momentum distribution width $f_{w}$, also measured in lattice sites, for the same situations is shown in (c) and (d), respectively. The dashed curves present in all plots are the fitted smooth "box" or "well" functions to the data.

which have been induced during the transition. These are visible as the oscillatory behavior of the eigenvalues seen in the latter section of the profile in Fig. 7. Their presence is consistent with the fact that larger systems have more numerous and closely spaced low-lying excitations; however, despite this the oscillations have only a small amplitude and so do not destroy the SF obtained at the end of the transition.

In order to examine the speed at which coherence is reestablished in the system during the latter half $\left(t>t_{c}\right)$ of the ramping profile the correlation length of the system must be computed over time. The correlation length is typically defined as the distance at which the off-diagonal elements of the one-particle density matrix become negligible [44]. For symmetrical systems, like those considered here, it is natural to measure this from the central site $m=25$. However, the inhomogeneity of the system, which results in the kind of correlations between spatially separated SF regions just discussed for Fig. 7(i), makes the determination of the correlation length ambiguous. Instead we choose to examine a cutoff length $\xi_{c}$ where the spatial correlations with the central site have a specific value $\left|\rho_{25,25+\xi_{c}}\right|=1 / e \approx 0.37$. This value is large enough that it corresponds to tracking a point on the central SF region and so can provide a relative measure of its size. The change in $\xi_{c}$ over time is plotted in Fig. 8(a). The same plot also shows the fitted curve whose function is that of a smooth "well" which is the reflection of the smooth "box" used earlier in Eq. (12) about the $t$ axis. The time parameters of this fit are very close to those of the resulting "well" for $J(t)$ generated from the $V_{0}(t)$ profile. The variation in $\xi_{c}$ over time demonstrates that it is capturing the essential changes in the central SF region, including the oscillations in its size at later times caused by excitations.

To investigate how much the presence of residual SF correlations affects the time dependence of $\xi_{c}$ the latter half $\left(t>t_{c}\right)$ of the same slow ramping profile was applied to the MI ground state of Fig. 4(c) found earlier for $U / 2 J=20$. In contrast to the state which is dynamically driven to the MI regime this ground state has virtually no off-diagonal correlations for any site. The change in $\xi_{c}$ of time for this case is shown in Fig. 8(b), and as before the fitted smooth well function is also plotted. These show that despite $\xi_{c}$ starting from a much smaller value for the MI ground state it still acquires the mean value of the final SF state on the same time scale as that of the dynamically driven state.

An important and experimentally motivated measure of the coherence of a state can be obtained from its momentum distribution function $p_{k}$. In experiments the interference pattern resulting from the state of the system is examined by allowing all the atoms within the lattice to expand freely for a short period of time and then measure the absorption $I(x)$ at points $x$ on a distant observation line. In the simplest model of this process one can neglect both the interactions between atoms during the expansion and the spatial dependence of the interference caused by the freely evolved Wannier function envelopes $w\left(x-x_{m}\right)$ [45]. This gives the generic features of the interference pattern at an observation point $x$ in terms of the path phases acquired by each site in a 1D lattice of phase coherent matter wave sources. In the far-field approximation the intensity $I(x)$ along the observation line is proportional to the momentum distribution $p_{k} \propto \sum_{m, n} \exp [i k(m-n)] \rho_{m, n}$ $[41,45]$.

The form of the momentum distribution is sufficiently well behaved that its width $f_{w}$ can be determined most easily by taking its standard deviation. The variation of $f_{w}$ in time for the full dynamics is shown in Fig. 8(c), along with the fitted smooth box function. The time parameters of this fit are again very similar to those of $J(t)$. As expected it is seen that $f_{w}$ increases in line with the decrease in off-diagonal correlations. The time profile of $f_{w}$ for the half-ramping of the MI ground state is shown in Fig. 8(d) and again confirms that the momentum distribution width of the SF is reestablished on approximately the same time scale as that of the dynamically driven state.

Finally we examine the speed at which the correlation cutoff length $\xi_{c}$ increases with time over the latter half of the slow ramping. At any given time $t$ the characteristic time scale at which single-atom hopping occurs is given by $\tau_{\text {tunnel }}(t)=\pi / 2 J(t)$ [46]. The simplest description of the growth of the central SF region is based on atoms at the edge of the system hopping towards the center. In this way correlations can be established over the whole lattice of $M$ sites [1]. An estimate for the overall time scale for this mechanism to occur is given by $t_{\text {restore }}=M \tau_{\text {tunnel }} / 2$ [47], which for the system and depths used here has a value $t_{\text {restore }} \approx 23 \mathrm{~ms}$. In Fig. 9 the speed $\partial \xi_{c} / \partial t$ obtained from the function fitting is plotted for (i) the full dynamics of the ramping and (ii) the half-ramping from the MI ground state. In line with the plots of $\xi_{c}$ in Figs. 8(a) and 8(b) we see that there is a time delay before there is a significant rate of change in $\xi_{c}$ for the MI ground state ramping. Since the restoration of correlations occurs over the same total time scale in both cases the peak in the correlation speed is higher for the MI ground state. In addition to these curves the characteristic tunneling speed 


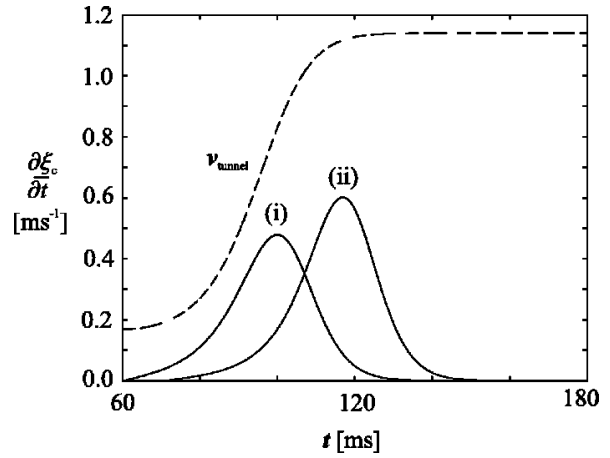

FIG. 9. A comparison between the speeds at which the correlation cutoff length $\xi_{c}$ changes in time for (i) the full slow ramping profile starting from the SF ground state and (ii) the last half of the ramping profile starting from the MI ground state at the bottom of the ramping. The time profile for the single-atom hopping speed $v_{\text {tunnel }}(t)$ is also shown. All speeds are expressed in lattice sites per ms.

$v_{\text {tunnel }}(t)=1 / \tau_{\text {tunnel }}(t)$ over the ramping is also shown, and most importantly we note that neither of the two correlation speeds (i) nor (ii) exceeds this curve. This confirms that the ramping applied is sufficiently slow that the propagation of the SF is dominated by single atom hopping.

\section{B. Fast dynamics}

\section{Profile for fast dynamics}

The time scale over which the slow ramping occurs is of the order of $60 \mathrm{~ms}$ and so greater than $t_{\text {restore }}$. Here we consider ramping occurring much more rapidly. Specifically we replace the latter part $\left(t>t_{c}\right)$ of the slow ramping profile with a linear ramping of the optical depth $V_{0}(t)$ from $V_{\mathrm{MI}}$ to $V_{\mathrm{SF}}$ as

$$
V_{0}(t)=V_{\mathrm{MI}}-\frac{\left(V_{\mathrm{MI}}-V_{\mathrm{SF}}\right)}{t_{\mathrm{ramp}}}\left(t-t_{c}\right),
$$

where $t$ runs from $t_{c}$ to $t_{c}+t_{\text {ramp }}$, and the total time of the ramping is $t_{\text {ramp. }}$ This gives a total ramping profile similar to that studied experimentally by Greiner et al. [1].

\section{Fast dynamics of the larger system: $M=49$}

For the fast dynamics we restrict our attention to the state that is dynamically driven to the MI regime by the slow ramping profile at $t=t_{c}$. A number of simulation runs were performed for total ramping times $t_{\text {ramp }}$ between $0.1 \mathrm{~ms}$ and $10 \mathrm{~ms}$, along with $t_{\text {ramp }}=0 \mathrm{~ms}$ which is equivalent to the initial state. The value of $\xi_{c}$ obtained at the end of each of the ramping times is plotted in Fig. 10(a). We see that there is a steady monotonic increase in the $\xi_{c}$ for ramping times $t_{\text {ramp }}$, except where it is broken by peaks and troughs which are the expected manifestations of the trapping used. In particular the trough centered around $t_{\text {ramp }} \approx 7 \mathrm{~ms}$ corresponds to the period of an oscillation with frequency $2 \omega$, where $\omega$ is the trapping frequency introduced in Sec. III B. On a similar basis the spikes which appear around $t_{\text {ramp }} \approx 1 \mathrm{~ms}$ can be seen to be a result of the excitation spectrum.
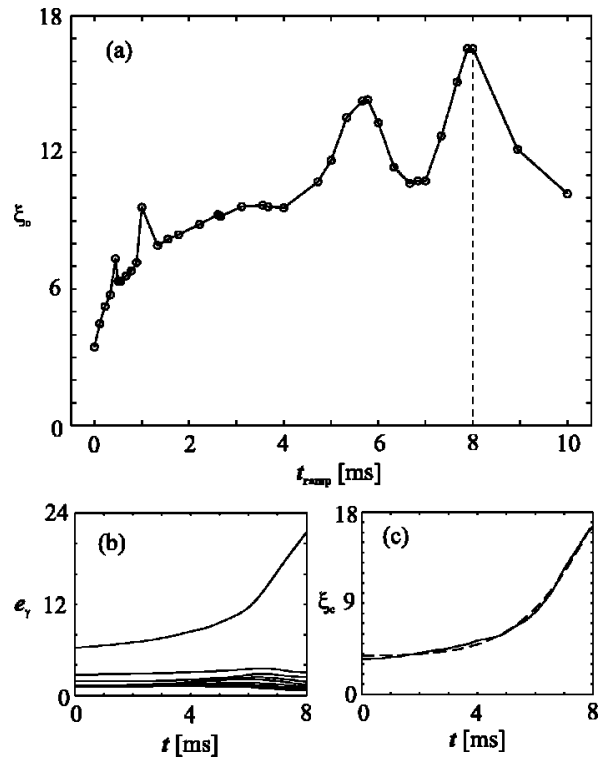

FIG. 10. Results for rapid dynamics, (a) the final correlation cutoff length $\xi_{c}$ obtained for different linear ramping times $t_{\text {ramp}}$, focusing on $t_{\text {ramp }}=8 \mathrm{~ms}$ we have (b) the spectrum of the oneparticle density matrix $e_{\gamma}$ showing only the largest ten eigenvalues, and (c) the variation in $\xi_{c}$ over the simulation run, along with a fitted smooth box function shown as the dashed curve.

We take a special interest in the ramping time $t_{\text {ramp }}$ $=8 \mathrm{~ms}$ where $\xi_{c}$ obtains its maximum value approximately equal to that of the SF ground state. The variation in time of the spectrum $e_{\gamma}$ of the one-particle density matrix and $\xi_{c}$ during this particular ramping simulation is given in Figs. 10 (b) and 10(c). A well-behaved monotonic increase in $\xi_{c}$ is observed which can be accurately fitted over the interval [0, 8] ms by a smooth box function, as used earlier. This again provides the basis for computing the speed $\partial \xi_{c} / \partial t$ at which the correlation cutoff length $\xi_{c}$ is increasing over the ramping and is shown in Fig. 11(b) along with that of the characteristic tunneling speed $v_{\text {tunnel }}(t)$ for the rapid ramping profile. Unlike the similar comparison for the slow dynamics we see here that after approximately $3 \mathrm{~ms} \xi_{c}$ is increasing in time much more rapidly than the single-site tunneling speed $v_{\text {tunnel }}$ alone can account for. Indeed by the end of the ramping $\partial \xi_{c} / \partial t$ is almost 3 times that of the maximum tunneling speed. This is a clear indication that single-atom hopping is not adequate to describe the growth of the central SF region for such rapid dynamics. Instead the specific form and contributions of higher-order correlation functions must play a crucial role.

To draw direct comparisons with the results of Greiner et al. [1] on the restoration of coherence we also plot the momentum distribution width $f_{w}$ obtained from the one-particle density matrix at the end of each ramping in Fig. 11(a). The data points for this quantity show a pronounced trend, without any of the large-scale variations seen in $\xi_{c}$ caused by the trapping [41]. The decrease of $f_{w}$ with increasing $t_{\text {ramp }}$ fits well to a double-exponential decay curve of the form

$$
f_{w}\left(t_{\mathrm{ramp}}\right)=A_{1} e^{-t_{\mathrm{ramp}} / \tau_{1}}+A_{2} e^{-t_{\mathrm{ramp}} / \tau_{2}}+C,
$$

where $\tau_{1}, \tau_{2}$ are the characteristic decay times, $A_{1}, A_{2}$ are coefficients, and $C$ is a constant. Most notably the exact 


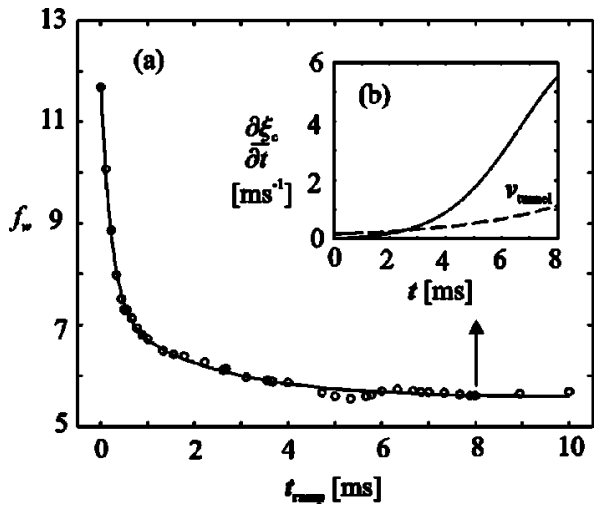

FIG. 11. Rapid ramping of an optical lattice from the MI $(U / 2 J=20)$ to the $\mathrm{SF}(U / 2 J=2)$ regime for $N=40$ atoms in $M$ $=49$ lattice sites superimposed by a magnetic trapping potential. The width of the central interference fringe $f_{w}$ as a function of the ramping time $t_{\text {ramp }}$ is shown in (a). The solid curve is a fit using a double-exponential decay $\left(\tau_{1}=0.22 \mathrm{~ms}, \tau_{2}=2.14 \mathrm{~ms}\right.$ ) (cf. [1]). In (b) the rate of change of the correlation cutoff length $\xi_{c}$ is shown for the ramping performed with $t_{\text {ramp }}=8 \mathrm{~ms}$, along with the profile for the characteristic tunneling speed $v_{\text {tunnel }}(t)$ for the ramping. Both are plotted in units of lattice sites per ms.

same functional form was found to fit the experimental data in [1]. Since their experiment was conducted for a 3D lattice, along with a larger mean occupancy and a deeper ramping profile, exact agreement for the time parameters of this fit is not expected. However, we do note that the ratio of the decay times used for their fit and ours are both $\tau_{2} / \tau_{1} \approx 10$. Similarly we can make the same observation as made in [1] that the momentum distribution width $f_{w}$ has returned to its steady-state value within a time scale approximately of order $\tau_{\text {tunnel. }}$. This is much shorter than the expected time $t_{\text {restore }}$ required for coherence to spread over the whole lattice of $M$ sites via single-atom hopping. This confirms that the restoration of coherence as seen in the experiment is accurately described by the BHM.

\section{Validity of the simulation for fast dynamics}

The simulations performed here assume that the dynamics of the atoms is described by the lowest Bloch band of the optical lattice. This assumption holds if the typical frequency $f \approx 1 / t_{\text {ramp }}$ of the ramp in $U$ and $J$ obeys $f \ll \nu$, where $\nu=\sqrt{4 E_{R} V_{0}} / 2 \pi$ is the harmonic approximation of the excitation frequency to the first excited Bloch band [15]. The shortest ramping time we considered is $t_{\mathrm{ramp}}=0.1 \mathrm{~ms}$, while $1 / \nu=0.05 \mathrm{~ms}$ for the lattice on average over the ramping. Because the condition $f \ll \nu$ is not fulfilled, we numerically calculated the probability of exciting a single particle, when initially prepared in the lowest Bloch band and located at the central site of the lattice, during the ramping above as a function of $t_{\text {ramp }}$. We find that the time evolution is well approximated by the adiabatic time evolution for $t_{\text {ramp }}>0.05 \mathrm{~ms}$ and that it changes to being sudden for $t_{\text {ramp }}<0.005 \mathrm{~ms}$. Therefore we expect only a small influence to the form of the curve in Fig. 11(a) between the points at $t_{\text {ramp }}=0 \mathrm{~ms}$ and $t_{\text {ramp }}=0.1 \mathrm{~ms}$ due to higher band excitations which is not resolved in the experiments [1].

\section{CONCLUSION}

In these studies we have established the accuracy and applicability of the TEBD algorithm to the BHM, for both the computation of ground states and its dynamics. We have then applied this method to systems of a size equivalent to those studied in experiments and in the presence of a trapping potential. In particular we have examined the nature and speed in which coherence is reestablished within the system for both slow and rapid dynamics which cross the SF-MI transition. Our results indicate that for slow ramping of the lattice depth the SF growth is consistent with single atom hopping as might naively be expected. However, for very rapid ramping of the lattice depth we find that the SF growth is much greater than can be explained by this mechanism alone and so points to the importance of higher-order correlation functions. We made direct comparisons between our simulation results for the momentum distribution width $f_{w}$ during rapid ramping and the experimental results obtained by Greiner et al. [1] and found that the reduction in $f_{w}$ with the ramping time follows precisely the same functional form as their data, despite a number of significant differences in the systems analyzed. Perhaps most fundamentally we have shown that the results obtained in [1] for the rapid restoration of coherence are consistent and explicable within the BHM alone and are present even in 1D systems. Finally, we note that a detailed knowledge of the correlations of atoms in different sites and the particle number fluctuations as provided by our numerical calculations are important for utilizing the MI state in a number of applications [6-14].

\section{ACKNOWLEDGEMNTS}

D.J. acknowledges useful discussions with Guifré Vidal and the hospitality of Caltech. This work was supported by EPSRC (UK).

\section{APPENDIX A: 1D OPTICAL LATTICES}

The starting point for our physical model is the Hamiltonian for weakly interacting bosonic atoms in an external trapping potential

$$
\begin{aligned}
H= & \int d \mathbf{r} \psi^{\dagger}(\mathbf{r})\left(-\frac{1}{2 m_{A}} \nabla^{2}+V_{0}(\mathbf{r})+V_{T}(\mathbf{r})\right) \psi(\mathbf{r}) \\
& +\frac{1}{2} \frac{4 \pi a_{s}}{m_{A}} \int d \mathbf{r} \psi^{\dagger}(\mathbf{r}) \psi^{\dagger}(\mathbf{r}) \psi(\mathbf{r}) \psi(\mathbf{r}),
\end{aligned}
$$

with $\psi(\mathbf{r})$ the bosonic field operator for atoms in a given internal atomic state, $V_{0}(\mathbf{r})$ is the optical lattice potential, and $V_{T}(\mathbf{r})$ describes a slowly varying additional external trapping potential such as that created by magnetic fields. The interaction between the atoms is modeled by a contact potential with $s$-wave scattering length $a_{s}$ and $m_{A}$ is the mass of the atoms.

We assume the optical lattice potential to have the form $V_{0}(\mathbf{r})=\sum_{j=1}^{3} V_{j 0} \sin ^{2}\left(k r_{j}\right)$ with wave number $k=2 \pi / \lambda$ and $\lambda$ the wavelength of the laser light yielding a lattice period $a$ $=\lambda / 2$. The spatial coordinate is denoted by $\mathbf{r}=\left(r_{1}, r_{2}, r_{3}\right)$. 
This lattice potential can be realized by interfering three pairs of counterpropagating laser beams from three orthogonal directions. The height of the potential $V_{j 0}$ is proportional to the intensity of the lasers in the $j$ th pair of laser beams. We assume the intensity to be very large in the $r_{2}$ and $r_{3}$ directions so that the atoms do not tunnel in either of these directions. Hence their motion is restricted to the $r_{1} \equiv x$ direction and this optical lattice setup thus allows the creation of effective 1D systems. The resulting atomic pipelines [2,10,23] are well isolated from each other and we can thus restrict our considerations to just one of them.

The center position of the lattice site $m$ of this 1D system is given by $x_{m}=m a$, and so a particle occupying the lowest Bloch band which is localized at this site is described by the wave function $\phi_{m}(\mathbf{r})=w_{1}\left(x-x_{m}\right) w_{2}\left(r_{2}\right) w_{3}\left(r_{3}\right)$, where $w_{j}$ are the Wannier functions of the lowest Bloch band [15] in the $j$ th direction. By neglecting all excitations to higher bands and expanding the bosonic field operators into the mode functions $\phi_{m}(\mathbf{r})$ the Hamiltonian $H$ reduces to the 1D BoseHubbard model [15] given in Eq. (1) Sec. II A. The parameter $U$ of the BHM is given by $U=4 \pi a_{s} \int d \mathbf{r}\left|\phi_{m}(\mathbf{r})\right|^{4} / m_{A}$ and corresponds to the strength of the on-site repulsion of two atoms occupying the same lattice site $m$. The hopping matrix element $J$ between adjacent sites $m$ and $m+1$ is given by

$J=-\int d x w_{1}\left(x-x_{m}\right)\left(-\frac{1}{2 m_{A}} \frac{d^{2}}{d x^{2}}+V_{0} \sin ^{2}(k x)\right) w_{1}\left(x-x_{m+1}\right)$.

The numerical values for $U$ and $J$ for different depths of the optical lattice $V_{0} \equiv V_{10}$ can be found in [15].

\section{APPENDIX B: MF APPROXIMATION AND GUTZWILLER} ANSATZ

The presence of a trapping potential causes the system to become inhomogeneous allowing the coexistence of spatially separated SF and MI regions [15,37-43]. Now each lattice site has a local chemical potential $\mu_{m}$, and together with the overall $U / 2 J$ parameter for the system this gives a phase diagram coordinate $\mathbf{p}=\left(2 J / U, \mu_{m} / U\right)$. The MF determination of which regime a given lattice site lies in is then based on where precisely $\mathbf{p}$ resides in the homogeneous BHM phase diagram $[5,41,48]$.

The MF calculations performed utilized the standard decoupling of the hopping term $[18,33]$

$$
b_{m}^{\dagger} b_{m+1}=b_{m}^{\dagger}\left\langle b_{m+1}\right\rangle+\left\langle b_{m}^{\dagger}\right\rangle b_{m+1}-\left\langle b_{m}^{\dagger}\right\rangle\left\langle b_{m+1}\right\rangle,
$$

which decompose the BHM Hamiltonian in Eq. (1) into a set of $M$ single site Hamiltonians $H_{m}$. Specifically the single site $H_{m}$ has the form

$$
H_{m}=-2 J\left(\phi_{m} b_{m}^{\dagger}+\text { h.c. }\right)-2\left|\phi_{m}\right|^{2}-\mu_{m} b_{m}^{\dagger} b_{m}+\frac{U}{2} b_{m}^{\dagger} b_{m}^{\dagger} b_{m} b_{m},
$$

for each lattice site $m$, dependent on the sites superfluid order parameter $\phi_{m}=\left\langle b_{m}\right\rangle$, which is assumed to vary slowly over the system, and its local chemical potential $\mu_{m}$. The MF ground state is then a product state over sites $\left|\psi_{\mathrm{MF}}\right\rangle$ $=\prod_{m=1}^{M}\left|\psi_{0}^{m}\right\rangle$ determined by minimizing the complete set of $M$ site Hamiltonians $H_{m}$ with respect to the order parameters $\phi_{m}$ and then diagonalizing to extract eigenstate $\left|\psi_{0}^{m}\right\rangle$ with the smallest eigenvalue for each site. Given that each $\left|\psi_{0}^{m}\right\rangle$ $=\Sigma_{n_{m}} c_{n_{m}}\left|n_{m}\right\rangle$ this procedure is equivalent to approximation methods based on the Gutzwiller ansatz $[5,32]$ and has been applied successfully in modeling the qualitative behavior of BHM phase diagram [48].
[1] M. Greiner, O. Mandel, T. Esslinger, T. W. Hänsch, and I. Bloch, Nature (London) 415, 39 (2002).

[2] T. Støferle, H. Moritz, C. Schori, M. Køhl, and T. Esslinger, Phys. Rev. Lett. 92, 130403 (2004).

[3] S. Peil, J. V. Porto, B. L. Tolra, J. M. Obrecht, B. E. King, M. Subbotin, S. L. Rolston, and W. D. Phillips, Phys. Rev. A 67, 051603 (2003).

[4] S. E. Sklarz, I. Friedler, D. J. Tannor, Y. B. Band, and C. J. Williams, Phys. Rev. A 66, 053620 (2002).

[5] M. P. A. Fisher, P. B. Weichman, G. Grinstein, and D. S. Fisher, Phys. Rev. B 40, 546 (1989).

[6] D. Jaksch, H. J. Briegel, J. I. Cirac, C. W. Gardiner, and P. Zoller, Phys. Rev. Lett. 82, 1975 (1999).

[7] H. J. Briegel, T. Calarco, D. Jaksch, J. I. Cirac, and P. Zoller, J. Mod. Opt. 47, 415 (2000).

[8] J. K. Pachos and P. L. Knight, Phys. Rev. Lett. 91, 107902 (2003).

[9] O. Mandel, M. Greiner, A. Widera, T. Rom, T. W. Hänsch, and I. Bloch, Nature (London) 425, 937 (2003).

[10] U. Dorner, P. Fedichev, D. Jaksch, M. Lewenstein, and P. Zoller, Phys. Rev. Lett. 91, 073601 (2003).
[11] G. K. Brennen, C. M. Caves, P. S. Jessen, and I. H. Deutsch, Phys. Rev. Lett. 82, 1060 (1999).

[12] D. Jaksch, J. I. Cirac, P. Zoller, S. L. Rolston, R. Cote, and M. D. Lukin, Phys. Rev. Lett. 85, 2208 (2000).

[13] E. Jane, G. Vidal, W. Dür, P. Zoller, and J. I. Cirac, Quantum Inf. Comput. 3, 15 (2003).

[14] A. Sörensen and K. Mölmer, Phys. Rev. Lett. 83, 2274 (1999).

[15] D. Jaksch, C. Bruder, J. I. Cirac, C. W. Gardiner, and P. Zoller, Phys. Rev. Lett. 81, 3108 (1998).

[16] H. P. Büchler, G. Blatter, and W. Zwerger, Phys. Rev. Lett. 90, 130401 (2003).

[17] D. Jaksch, V. Venturi, J. I. Cirac, C. J. Williams, and P. Zoller, Phys. Rev. Lett. 89, 040402 (2002).

[18] K. Sheshadri, H. R. Krishnamurthy, R. Pandit, and T. V. Ramakrishnan, Europhys. Lett. 22, 257 (1993).

[19] J. K. Freericks and H. Monien, Europhys. Lett. 26, 545 (1994).

[20] G. Vidal, Phys. Rev. Lett. 91, 147902 (2003).

[21] G. Vidal, Phys. Rev. Lett. 93, 040502 (2004).

[22] A. J. Daley, C. Kollath, U. Schollwöck, and G. Vidal, J. Stat. Mech: Theor. Exp P04005 (2004). 
[23] B. Paredes, A. Widera, V. Murg, O. Mandel, S. Fölling, I. Cirac, G. Shlyapnikov, T. W. Hänsch, and I. Bloch, Nature (London) 429, 277 (2004).

[24] S. R. White, Phys. Rev. Lett. 69, 2863 (1992).

[25] S. R. White, Phys. Rev. B 48, 10345 (1993).

[26] E. Jeckelmann, Phys. Rev. B 66, 045114 (2002).

[27] M. A. Cazalilla and J. B. Marston, Phys. Rev. Lett. 88, 256403 (2002); 91, 049702 (2003).

[28] H. G. Luo, T. Xiang, and X. Q. Wang, Phys. Rev. Lett. 91, 049701 (2003).

[29] S. R. White and A. E. Feiguin, e-print cond-mat/0403310.

[30] M. Fannes, B. Nachtergaele, and R. F. Werner, Commun. Math. Phys. 144, 3 (1992).

[31] S. Östlund and S. Rommer, Phys. Rev. Lett. 75, 3537 (1995).

[32] W. Krauth, M. Caffarel, and J. P. Bouchaud, Phys. Rev. B 45, 3137 (1992).

[33] D. van Oosten, P. van der Straten, and H. T. C. Stoof, Phys. Rev. A 63, 053601 (2001).

[34] M. Suzuki, Phys. Lett. A 146, 6 (1990); J. Math. Phys. 32, 2 (1991).

[35] M. Zwolak and G. Vidal, e-print cond-mat/0406440.

[36] F. Verstraete, J. J. Garía-Ripoll, and J. I. Cirac, e-print condmat/0406426.

[37] C. Schroll, F. Marquardt, and C. Bruder, e-print cond-mat/ 0404576.
[38] G. G. Batrouni, V. Rousseau, R. T. Scalettar, M. Rigol, A. Muramatsu, P. J. H. Denteneer, and M. Troyer, Phys. Rev. Lett. 89, 117203 (2002).

[39] S. Bergkvist, P. Henelius, and A. Rosengren, e-print cond-mat/ 0404395.

[40] C. Kollath, U. Schollwöck, J. von Delft, and W. Zwerger, Phys. Rev. A 69, 031601(R) (2004).

[41] V. A. Kashurnikov, N. V. Prokof'ev, and B. V. Svistunov, Phys. Rev. A 66, 031601 (2002).

[42] S. Wessel, F. Alet, M. Troyer, and G. G. Batrouni, e-print cond-mat/0404552.

[43] L. Pollet, S. Rombouts, K. Heyde, and J. Dukelsky, Phys. Rev. A 69, 043601 (2004).

[44] L. Pitaevskii and S. Stringari, Bose-Einstein Condensation (Oxford University Press, Oxford, 2003).

[45] R. Roth and K. Burnett, Phys. Rev. A 67, 031602 (2003).

[46] We define the time scale $\tau_{\text {tunnel }}=\pi / 2 J$. This is physically motivated by considering the time required for one atom, within a double-well potential, to hop from one well to another, given a tunneling matrix element $J$.

[47] To define a time scale for hopping across half the lattice, when $J$ varies in time, some appropriate value must be chosen to represent its typical value during the ramping.

[48] S. Sachdev, Quantum Phase Transitions (Cambridge University Press, Cambridge, England, 2001). 